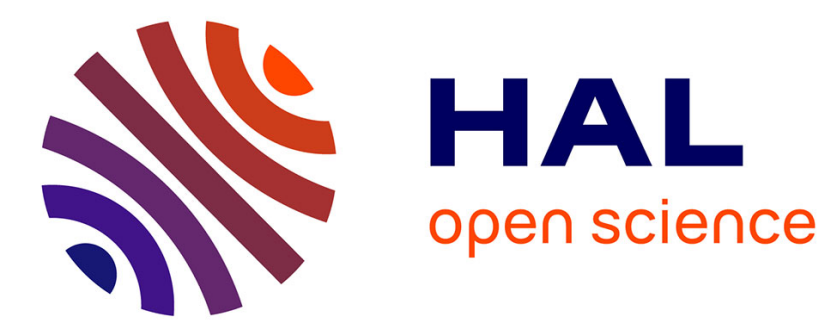

\title{
Composition of Titan's Atmosphere
}

\author{
Véronique Vuitton
}

\section{To cite this version:}

Véronique Vuitton. Composition of Titan's Atmosphere. David Alderton; Scott A. Elias. Encyclopedia of Geology (Second Edition), Elsevier, pp.217 - 230, 2021, 978-0-08-102909-1. 10.1016/b978-0-08102908-4.00064-3 . hal-03118616

\section{HAL Id: hal-03118616 https://hal.science/hal-03118616}

Submitted on 5 Nov 2021

HAL is a multi-disciplinary open access archive for the deposit and dissemination of scientific research documents, whether they are published or not. The documents may come from teaching and research institutions in France or abroad, or from public or private research centers.
L'archive ouverte pluridisciplinaire HAL, est destinée au dépôt et à la diffusion de documents scientifiques de niveau recherche, publiés ou non, émanant des établissements d'enseignement et de recherche français ou étrangers, des laboratoires publics ou privés. 


\section{Composition of Titan's Atmosphere}

\section{Véronique Vuitton}

veronique.vuitton@univ-grenoble-alpes.fr

Institut de Planétologie et d'Astrophysique de Grenoble Université Grenoble Alpes, Grenoble, France

\section{Introduction}

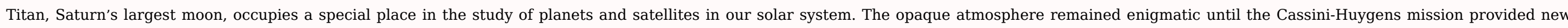

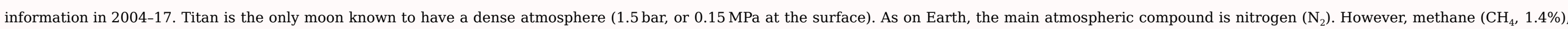

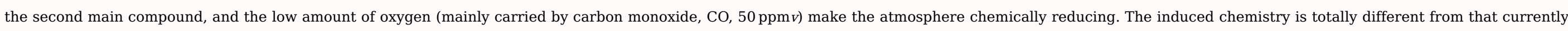

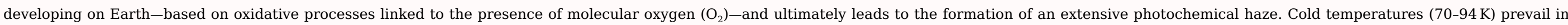

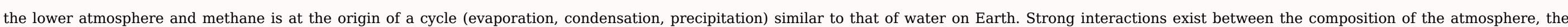

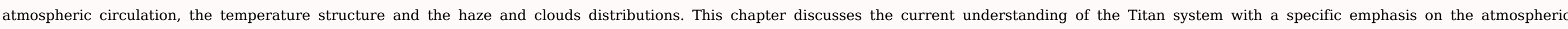
composition and its seasonal variations.

\section{Discovery and Exploration}

\section{Pre-Cassini Background}

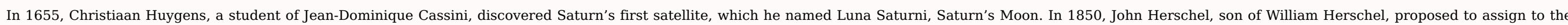

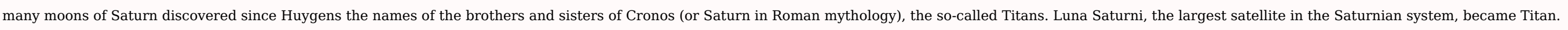

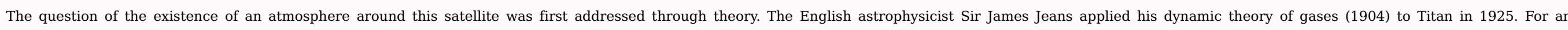

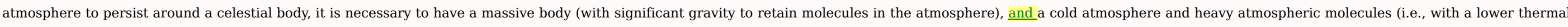

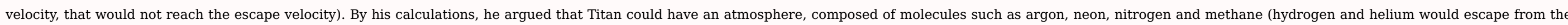
atmosphere).

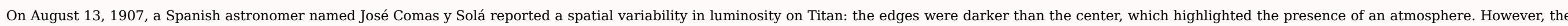

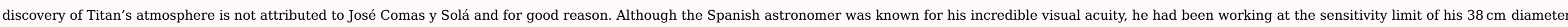
instrument and no other contemporary astronomer since has been able to see this phenomenon.

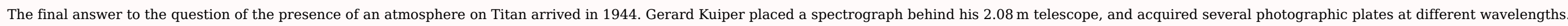

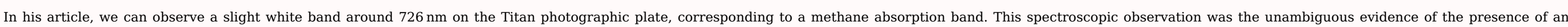
atmosphere on Titan.

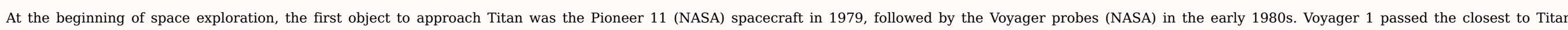

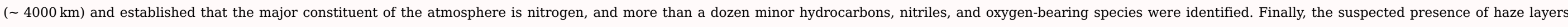

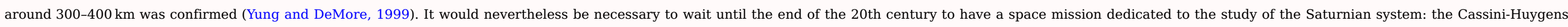
mission.

\section{The Cassini-Huygens Mission}




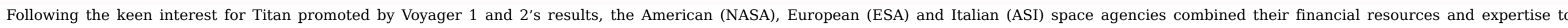

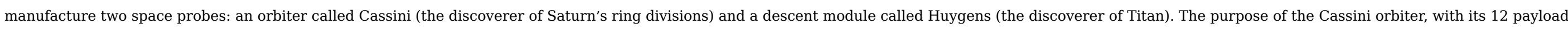

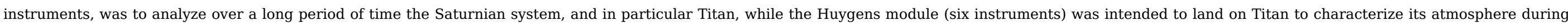
the descent, and its surface following landing.

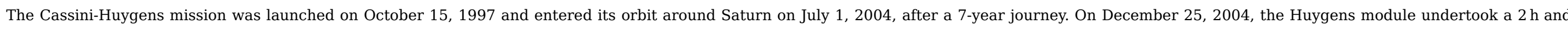

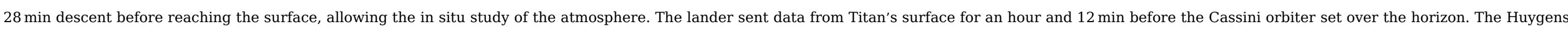

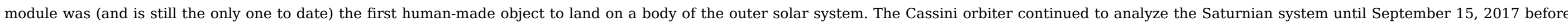

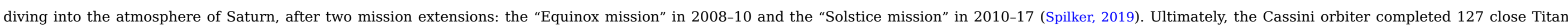
flybys and analyzed the moon for over half a Titan year, between mid-north winter and early north summer (Fig. 1).

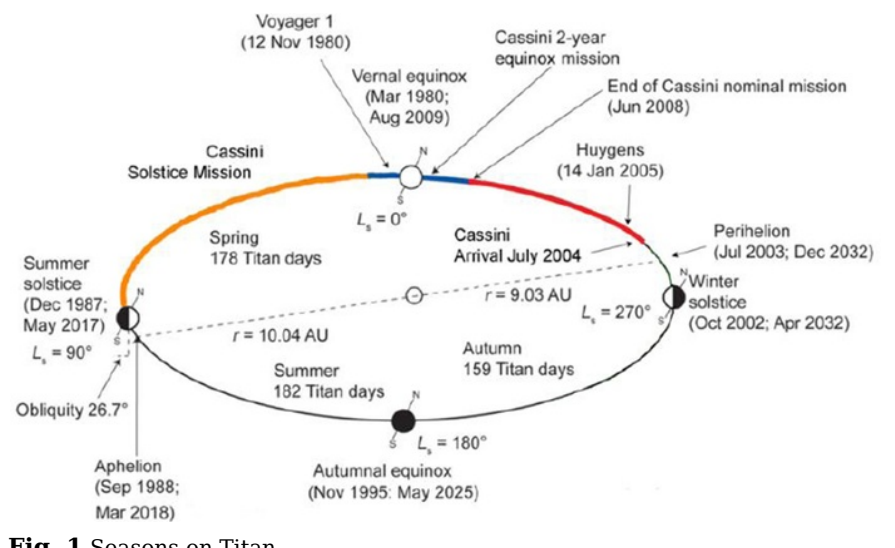

Fig. 1 Seasons on Titan.

Reproduced from Leary JC et al. (2008) Titan Explorer: Flagship Mission Study.

\section{alt-text: Fig. 1}

\section{General Description}

\section{Orbit}

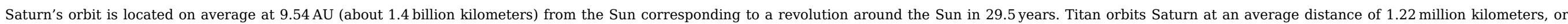

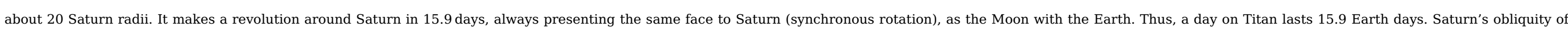

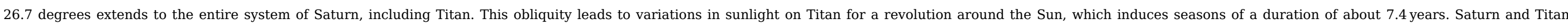
orbital data are given in Table 1.

Table 1 Orbital parameters of Saturn and Titan.

\begin{tabular}{|l|l|}
\hline alt-text: Table 1 \\
\hline Saturn's orbit & \\
\hline Orbital period & 29.5 years \\
\hline Semi-major axis & $1.43 \times 10^{9} \mathrm{~km}(9.58 \mathrm{AU})$ \\
\hline Eccentricity & 0.0565 \\
\hline
\end{tabular}


Titan's orbit

\begin{tabular}{l|l}
\hline Orbital period & 15.9 days \\
\hline Semi-major axis & $1.22 \times 10^{6} \mathrm{~km}\left(20.3 \mathrm{R}_{\mathrm{S}}\right)$ \\
\hline Eccentricity & 0.0288 \\
\hline Axial tilt & $\sim$ Zero
\end{tabular}

\section{Interior}

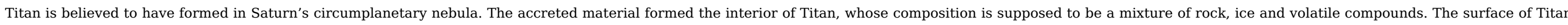

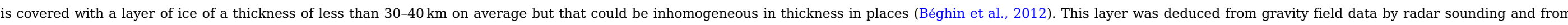

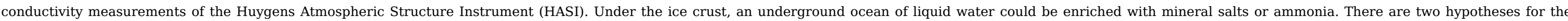

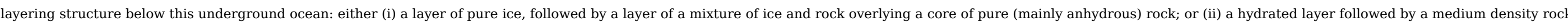

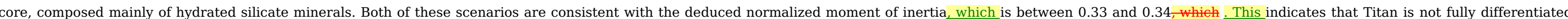
and does not have a large iron core (Fortes, 2012). Titan's physical data are given in Table 2 .

Table 2 Titan's physical characteristics.

\begin{tabular}{|l|l|}
\hline alt-text: Table 2 & \\
\hline Mean radius & $2574.73 \pm 0.09 \mathrm{~km}(0.404$ Earths $)$ \\
\hline Mass & $(1.3452 \pm 0.0002) \times 10^{23} \mathrm{~kg}(0.0225$ Earths $)$ \\
\hline Mean density & $1.8798 \pm 0.0044{\mathrm{~g} . \mathrm{cm}^{-3}}^{-3}$ \\
\hline Surface gravity & $1.352 \mathrm{~m} \mathrm{~s}^{-2}(0.138 \mathrm{~g})$ \\
\hline
\end{tabular}

\section{Surface}

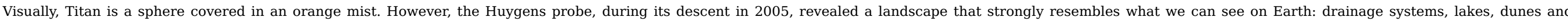

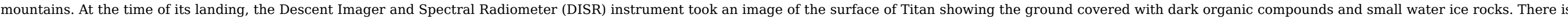

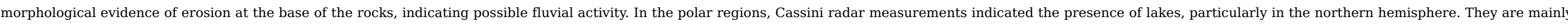

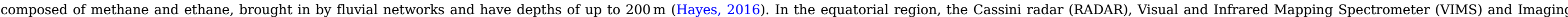

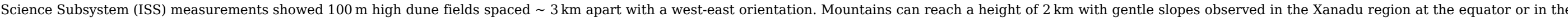

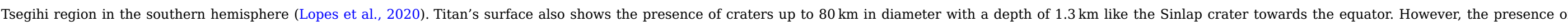
cryovolcanoes remains debated (Sotin et al., 2005).

\section{Atmosphere}

\section{Origin}

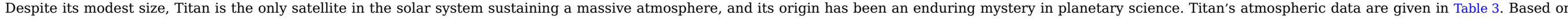

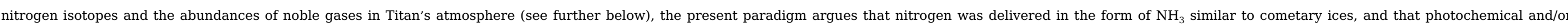

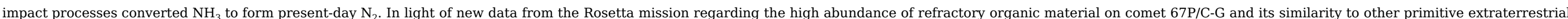


Table 3 Titan's atmospheric characteristics.

\begin{tabular}{|l|l|}
\hline alt-text: Table 3 & \\
\hline Surface temperature & $93.7 \mathrm{~K}\left(-179.5^{\circ} \mathrm{C}\right)$ \\
\hline Surface pressure & $146.7 \mathrm{kPa}(1.45 \mathrm{~atm})$ \\
\hline Stratospheric composition (by volume) & $98.4 \%$ \\
\hline Nitrogen $\left(\mathrm{N}_{2}\right)$ & $1.4 \%$ \\
\hline Methane $\left(\mathrm{CH}_{4}\right)$ & $0.2 \%$ \\
\hline Hydrogen $\left(\mathrm{H}_{2}\right)$ & \\
\hline
\end{tabular}

\section{Main vertical structure}

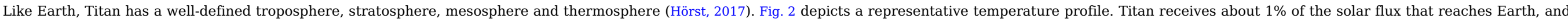

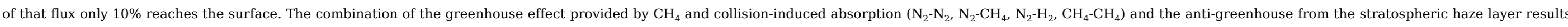

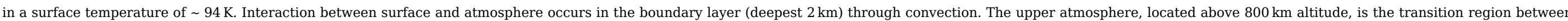

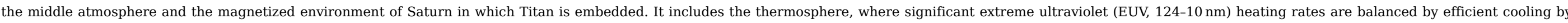

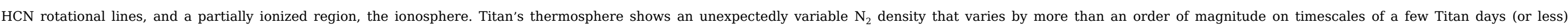

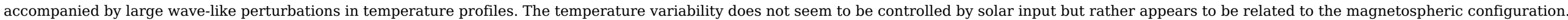

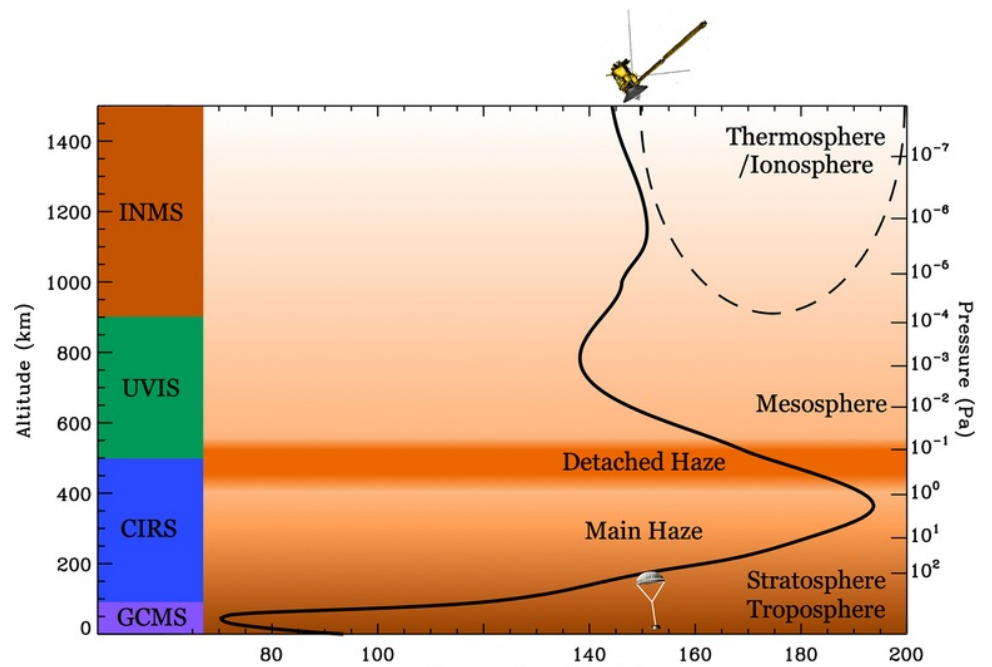

Fig. 2 Titan's atmospheric structure. The left side of the diagram shows the altitudes probed by different instruments onboard Cassini.

Copyright Sarah M. Hörst 2013.

alt-text: Fig. 2 


\section{Methane cycle}

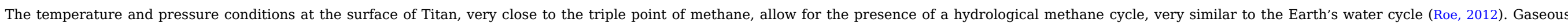

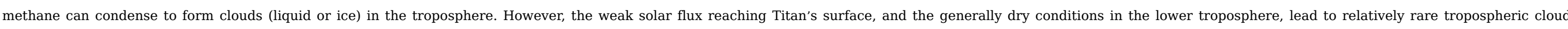

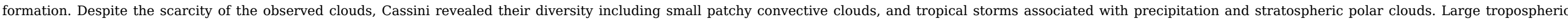

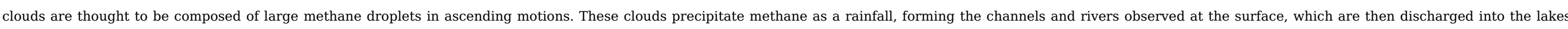
located mainly in the polar regions.

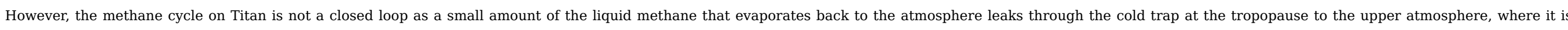

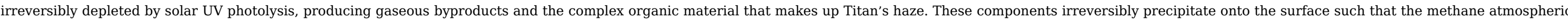

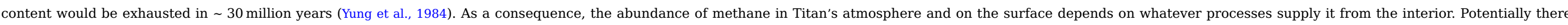
have been dry, methane-free epochs, as well as wet and rainy methane-rich periods.

\section{Complex Chemical Processes}

\section{Energy Sources}

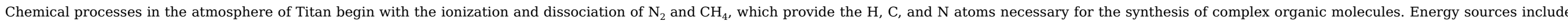

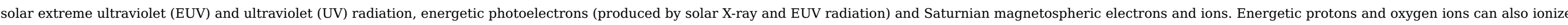

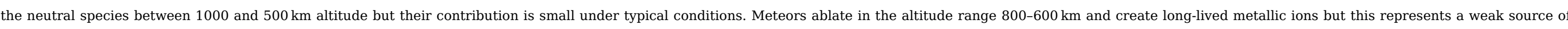

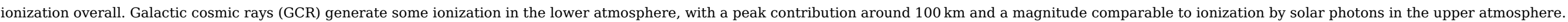

\section{Ionosphere}

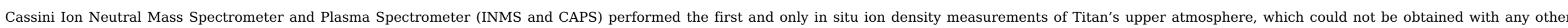

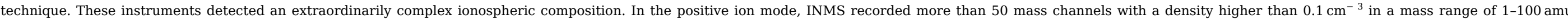

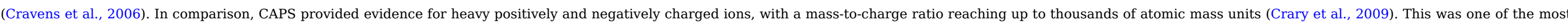

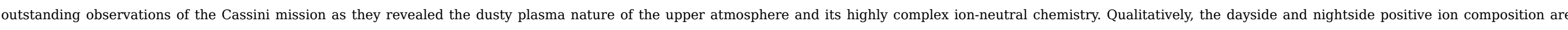

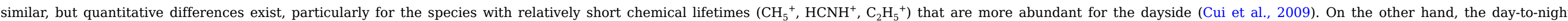

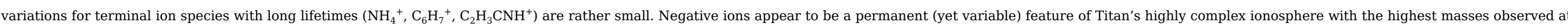
the lowest sampled altitudes $(950 \mathrm{~km})$.

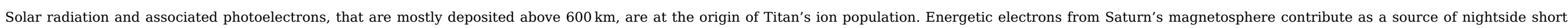

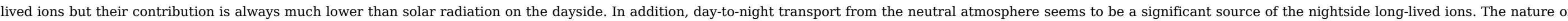

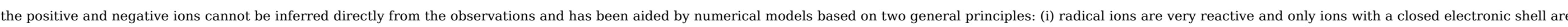

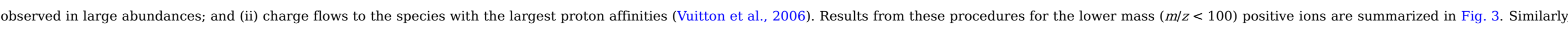

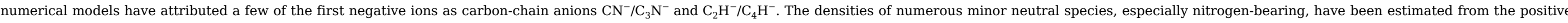

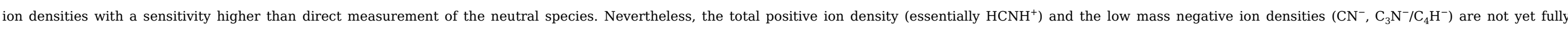
explained. 

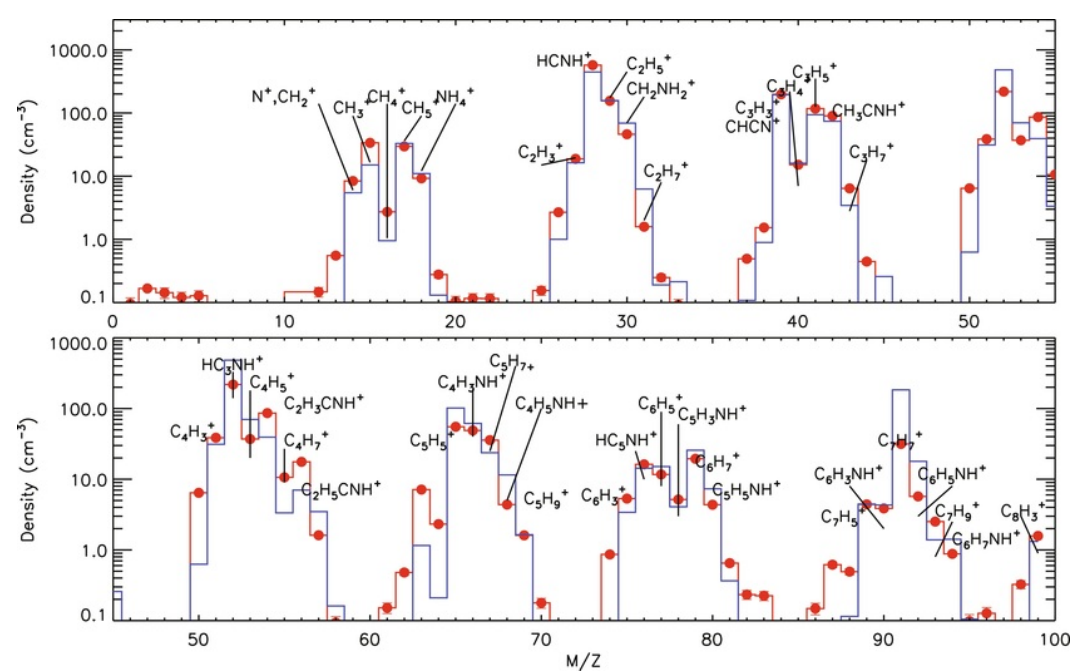

Fig. 3 Cassini/INMS (dots connected by red lines) and computed (blue lines) mass spectrum.

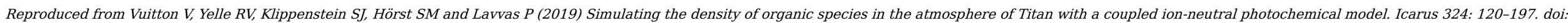
https://doi.org/10.1016/j.icarus.2018.06.013.

\section{alt-text: Fig. 3}

\section{Neutral Species}

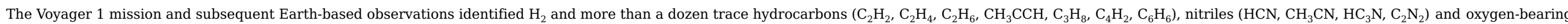

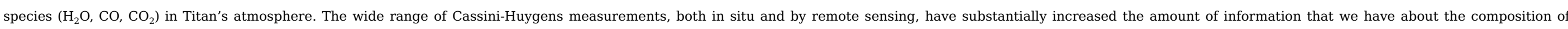

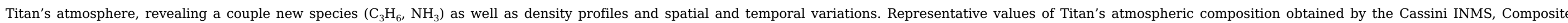

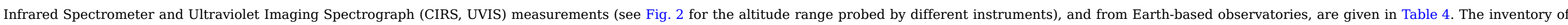

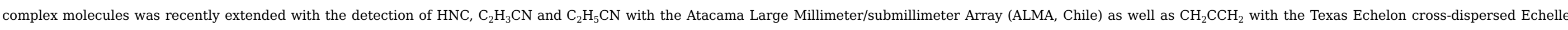
Spectrograph on the NASA Infrared Telescope Facility (TEXES/IRTF, Hawaii) but with limited spatial and vertical coverage.

Table 4 Composition of Titan's neutral atmosphere.

\section{alt-text: Table 4}

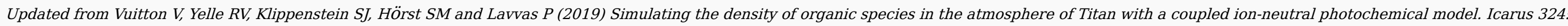
120-197, doi: https://doi.org/10.1016/j.icarus.2018.06.013.

\begin{tabular}{|c|c|c|c|c|c|}
\hline \multirow[t]{2}{*}{ Species } & \multicolumn{3}{|c|}{ Stratosphere } & \multirow{2}{*}{$\begin{array}{l}\text { Mesosphere } \\
\text { Cassini UVIS }\end{array}$} & \multirow{2}{*}{$\begin{array}{l}\text { Thermosphere } \\
\text { Cassini INMS }\end{array}$} \\
\hline & Ground based & ISO/Herschel & Cassini CIRS & & \\
\hline $\mathrm{H}_{2}$ (hydrogen) & & & $(9.6 \pm 2.4) \times 10^{-4}$ & & $(3.9 \pm 0.01) \times 10^{-3}$ \\
\hline $\mathrm{C}_{2} \mathrm{H}_{2}$ (acetylene) & & $(5.5 \pm 0.5) \times 10^{-6}$ & $2.97 \times 10^{-6}$ & $(5.9 \pm 0.6) \times 10^{-5}$ & $(3.1 \pm 1.1) \times 10^{-4}$ \\
\hline $\mathrm{C}_{2} \mathrm{H}_{4}$ (ethylene) & & $(1.2 \pm 0.3) \times 10^{-7}$ & $1.2 \times 10^{-7}$ & $(1.6 \pm 0.7) \times 10^{-6}$ & $(3.1 \pm 1.1) \times 10^{-4}$ \\
\hline $\mathrm{C}_{2} \mathrm{H}_{6}$ (ethane) & & $(2.0 \pm 0.8) \times 10^{-5}$ & $7.3 \times 10^{-6}$ & & $(7.3 \pm 2.6) \times 10^{-5}$ \\
\hline $\mathrm{CH}_{3} \mathrm{C}_{2} \mathrm{H}$ (propyne) & & $(1.2 \pm 0.4) \times 10^{-8}$ & $4.8 \times 10^{-9}$ & & $(1.4 \pm 0.9) \times 10^{-4}$ \\
\hline
\end{tabular}




\begin{tabular}{|c|c|c|c|c|c|}
\hline $\mathrm{CH}_{2} \mathrm{CCH}_{2}$ (propadiene) & $(6.9 \pm 0.8) \times 10^{-10}$ & & & & \\
\hline $\mathrm{C}_{3} \mathrm{H}_{6}$ (propene) & & & $(7 \pm 3) \times 10^{-9}$ & & $(2.3 \pm 0.2) \times 10^{-6}$ \\
\hline $\mathrm{C}_{3} \mathrm{H}_{8}$ (propane) & $(6.2 \pm 1.2) \times 10^{-7}$ & $(2.0 \pm 1.0) \times 10^{-7}$ & $4.5 \times 10^{-7}$ & & $<4.8 \times 10^{-5}$ \\
\hline $\mathrm{C}_{4} \mathrm{H}_{2}$ (diacetylene) & & $(2.0 \pm 0.5) \times 10^{-9}$ & $1.12 \times 10^{-9}$ & $(7.6 \pm 0.9) \times 10^{-7}$ & $(6.4 \pm 2.7) \times 10^{-5}$ \\
\hline $\mathrm{C}_{6} \mathrm{H}_{6}$ (benzene) & & $(4.0 \pm 3.0) \times 10^{-10}$ & $2.2 \times 10^{-10}$ & $(2.3 \pm 0.3) \times 10^{-7}$ & $(8.95 \pm 0.44) \times 10^{-7}$ \\
\hline HCN (hydrogen cyanide) & $5 \times 10^{-7}$ & $(3.0 \pm 0.5) \times 10^{-7}$ & $6.7 \times 10^{-8}$ & $(1.6 \pm 0.7) \times 10^{-5}$ & \\
\hline HNC (hydrogen isocyanide) & $(4.9 \pm 0.3) \times 10^{-9}$ & $(4.5 \pm 1.2) \times 10^{-9}$ & & & \\
\hline $\mathrm{HC}_{3} \mathrm{~N}$ (cyanoacetylene) & $(4.50 \pm 0.75) \times 10^{-9}$ & $(5.0 \pm 3.5) \times 10^{-10}$ & $2.8 \times 10^{-10}$ & $(2.4 \pm 0.3) \times 10^{-6}$ & $(3.2 \pm 0.7) \times 10^{-5}$ \\
\hline $\mathrm{CH}_{3} \mathrm{CN}$ (acetonitrile) & $(1.27 \pm 0.03) \times 10^{-8}$ & & $<1.1 \times 10^{-7}$ & & $(3.1 \pm 0.7) \times 10^{-5}$ \\
\hline $\mathrm{C}_{2} \mathrm{H}_{3} \mathrm{CN}$ (acrylonitrile) & $(3.03 \pm 0.29) \times 10^{-9}$ & & & & $<1.8 \times 10^{-5}$ \\
\hline $\mathrm{C}_{2} \mathrm{H}_{5} \mathrm{CN}$ (propionitrile) & $(7.37 \pm 0.32) \times 10^{-9}$ & & & & \\
\hline $\mathrm{C}_{2} \mathrm{~N}_{2}$ (cyanogen) & & & $9 \times 10^{-10}$ & & $(4.8 \pm 0.8) \times 10^{-5}$ \\
\hline $\mathrm{NH}_{3}$ (ammonia) & & $<1.9 \times 10^{-10}$ & $<1.3 \times 10^{-9}$ & & $(2.99 \pm 0.22) \times 10^{-5}$ \\
\hline $\mathrm{H}_{2} \mathrm{O}$ (water) & & $8 \times 10^{-9 / 7} \times 10^{-10}$ & $(4.5 \pm 1.5) \times 10^{-10}$ & & $<3.42 \times 10^{-6}$ \\
\hline CO (carbon monoxide) & $(4.96 \pm 0.18) \times 10^{-5}$ & $(4.0 \pm 5) \times 10^{-5}$ & $(4.7 \pm 0.8) \times 10^{-5}$ & & \\
\hline $\mathrm{CO}_{2}$ (carbon dioxide) & & $(2.0 \pm 0.2) \times 10^{-8}$ & $1.1 \times 10^{-8}$ & & $<8.49 \times 10^{-7}$ \\
\hline
\end{tabular}

The completeness in observational coverage varies substantially by molecule and we focus on the equatorial and mid-latitudes as the observations allow.

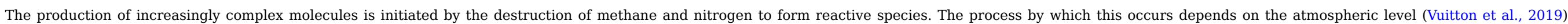

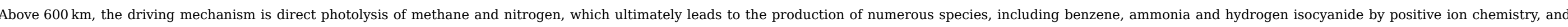

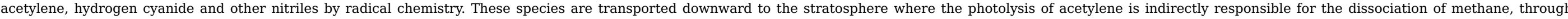

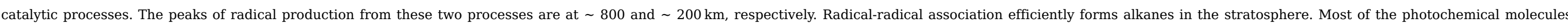
formed in the upper and middle atmosphere condense at the top of the troposphere, giving rise to vertical abundance profiles that have increasing volume mixing ratios with increasing altitude.

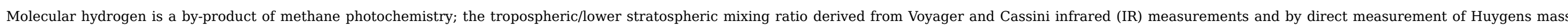

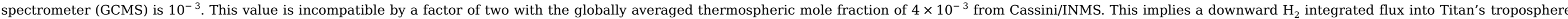
comparable to the $\mathrm{H}_{2}$ escape rate out of the atmosphere $\left(\sim 10^{28} \mathrm{H}_{2} \mathrm{~s}^{-1}\right)$

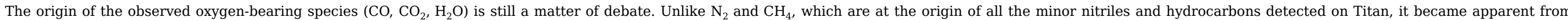

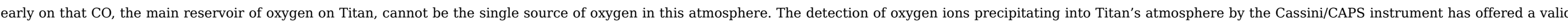

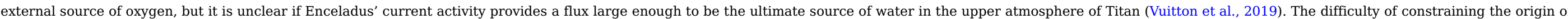

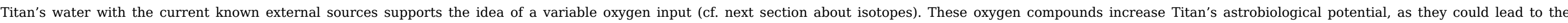
formation of amino acids and DNA nucleobases, as suggested from laboratory experiments that simulate Titan's chemistry (Hörst et al., 2012).

\section{Isotopes}

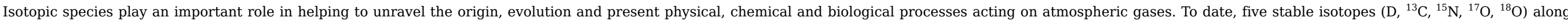
with radiogenic ${ }^{40} \mathrm{Ar}$ have been detected in Titan's atmosphere. The stable isotopes have been detected in a variety of compounds (see Table 5) whereas the noble gas ${ }^{40} \mathrm{Ar}$ was detected as a free atom. 
Table 5 Titan's isotopic ratios.

alt-text: Table 5

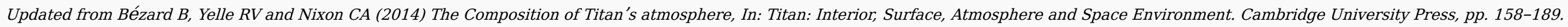

\begin{tabular}{|c|c|c|c|c|c|c|}
\hline \multirow[t]{2}{*}{ Species } & \multicolumn{2}{|c|}{ Sub-mm } & \multicolumn{2}{|c|}{ IR } & \multicolumn{2}{|c|}{ MS } \\
\hline & ALMA & Herschel SPIRE/PACS & IRTF TEXES/MPT Celeste & Cassini CIRS & Huygens GCMS & Cassini INMS \\
\hline
\end{tabular}

$\mathrm{D} / \mathrm{H}\left(1.56 \times 10^{-4}\right)$

$\mathrm{H}_{2}$

\begin{tabular}{|l|l|r|l|}
\hline $\mathrm{CH}_{4}$ & $(1.033 \pm 0.081) \times 10^{-4}$ & $(1.25 \pm 0.25) \times 10^{-4}$ & $(1.59 \pm 0.27) \times 10^{-4}$ \\
\hline $\mathrm{C}_{2} \mathrm{H}_{2}$ & & $(1.63 \pm 0.27) \times 10^{-4}$ \\
\hline $\mathrm{HCN}$ & $(2.5 \pm 0.2) \times 10^{-4}$ & $(2.09 \pm 0.45) \times 10^{-4}$ \\
\hline
\end{tabular}

${ }^{12} \mathrm{C} /{ }^{13} \mathrm{C}$ (89.3)

$\mathrm{CH}_{4}$

$\mathrm{C}_{2} \mathrm{H}_{2}$

$\mathrm{C}_{2} \mathrm{H}_{6}$

$\mathrm{C}_{4} \mathrm{H}_{2}$

HCN

$\mathrm{HC}_{3} \mathrm{~N}$

$\mathrm{CO}$

$\mathrm{CO}_{2}$

${ }^{14} \mathrm{~N} /{ }^{15} \mathrm{~N}$

$\mathrm{N}_{2}$

$\mathrm{HCN}$

$72.3 \pm 2.2$

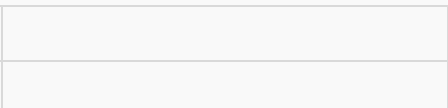

$(2.09 \pm 0.45) \times 10^{-4}$

$\mathrm{HC}_{3} \mathrm{~N}$

$67 \pm 14$

$86.5 \pm 7.9$

$91.1 \pm 1.4$

$88.5 \pm 1.4$

${ }^{16} \mathrm{O} /{ }^{17} \mathrm{O}(2680.6)$

\begin{tabular}{l|l} 
CO & $2917 \pm 359$
\end{tabular}

${ }^{16} \mathrm{O} /{ }^{18} \mathrm{O}$ (498.8)

\begin{tabular}{|l|l|l|l|l|}
\hline $\mathrm{CO}$ & $486 \pm 22$ & $380 \pm 60$ & $380 \pm 142$ \\
\hline $\mathrm{CO}_{2}$ & & & 380 \\
\hline
\end{tabular}

${ }^{36} \mathrm{Ar} /{ }^{40} \mathrm{Ar}$

$\mathrm{Ar}$

$84.8 \pm 3.2$

$89.8 \pm 7.3$

$90 \pm 8$

$75 \pm 12$

$79 \pm 17$

$84 \pm 17$

$(1.35 \pm 0.30) \times 10^{-4}$

Terrestrial values are enclosed in brackets.

D/H ratio 


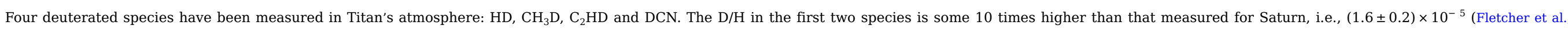

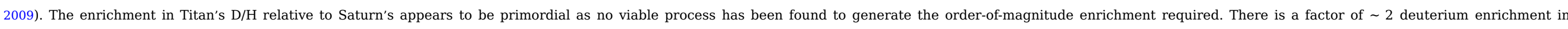

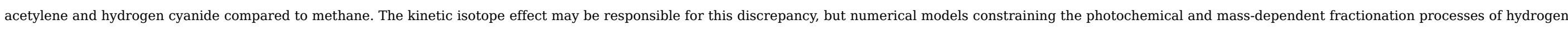
and deuterium on Titan remain to be developed.

\section{${ }^{12} \mathrm{C} /{ }^{3} \mathrm{C}$ ratio}

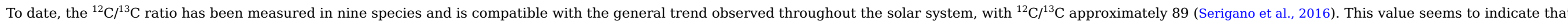
methane is being continuously replenished at present, otherwise it should have evolved from this presumed starting value through isotope-selective chemistry and photolysis.

\section{${ }^{14} \boldsymbol{N}{ }^{35} \boldsymbol{N}$ ratio}

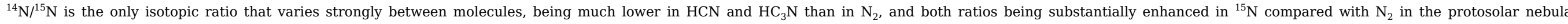

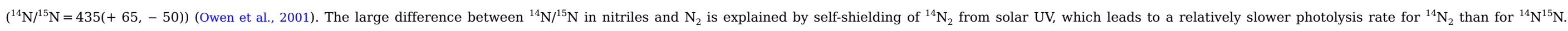

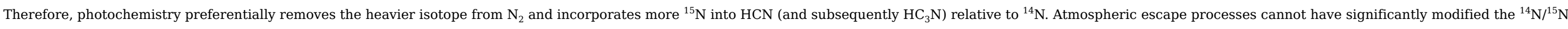

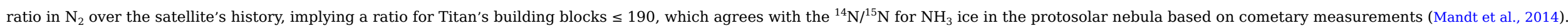

\section{${ }^{16} \boldsymbol{O} /{ }^{17} \boldsymbol{O} /{ }^{8} \mathrm{O}$ ratio}

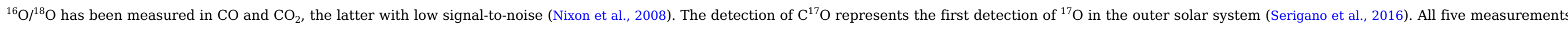

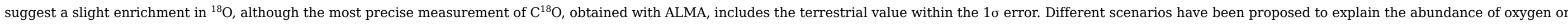

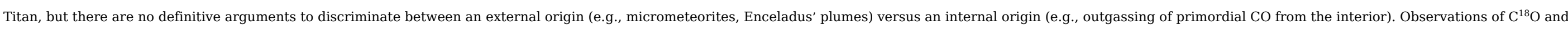

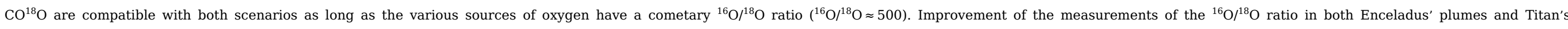
atmospheric $\mathrm{CO}_{2}$ could give a valuable constraint on the origin of oxygen in Titan's atmosphere.

\section{${ }^{36}$ Ar ${ }^{\mu 0}$ Ar ratio}

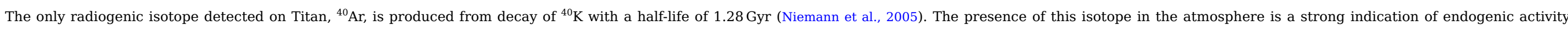
leading to the release of ${ }^{40} \mathrm{Ar}$ produced in the silicate fraction of the interior. This detection shows that trapping of highly volatile gases, including possibly $\mathrm{N}_{2}$ and CO, was possible during Titan's formation.

\section{Haze}

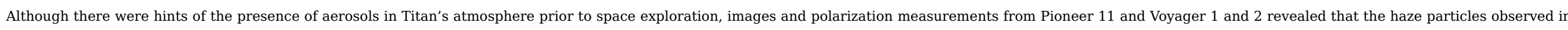

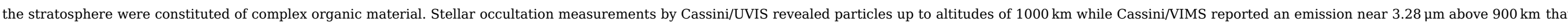

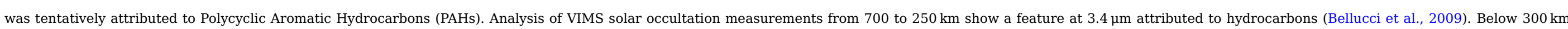

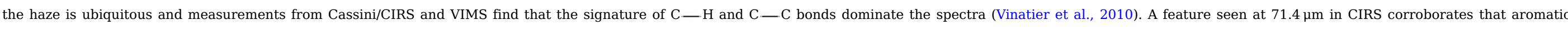

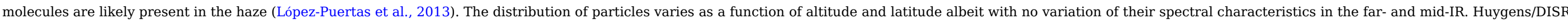

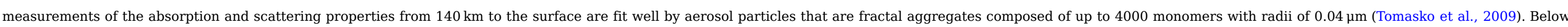

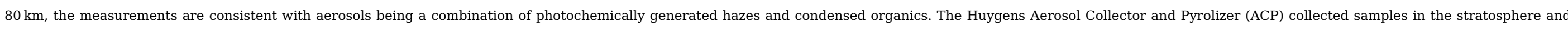

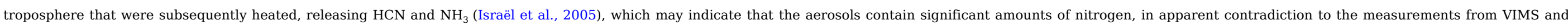
CIRS (Bellucci et al., 2009; Vinatier et al., 2010).

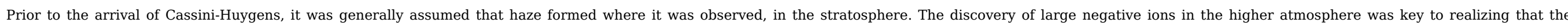

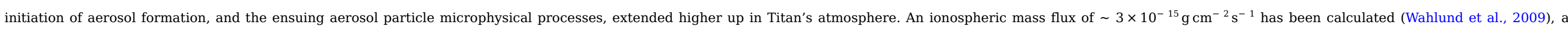

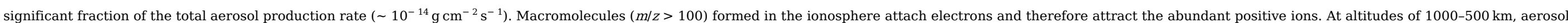




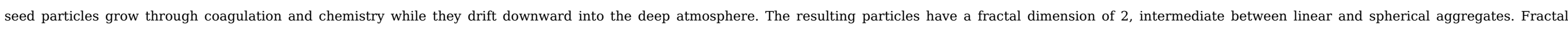

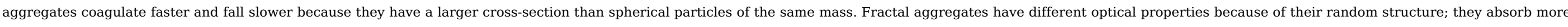

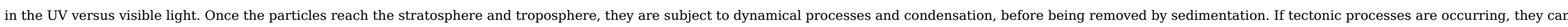
be mixed downwards into the interior, undergoing further change and oxidation in the aqueous environment.

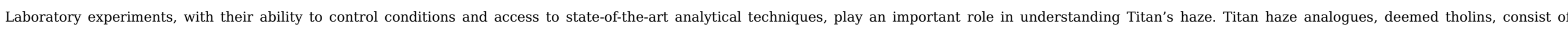

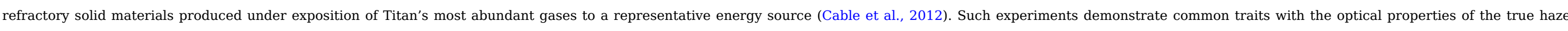

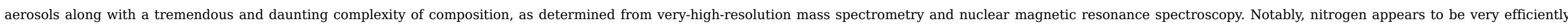
incorporated into the solid phase.

\section{Coupling Between Atmospheric Dynamics, Composition and Haze Distributions Atmospheric Dynamics}

\section{Meridional circulation}

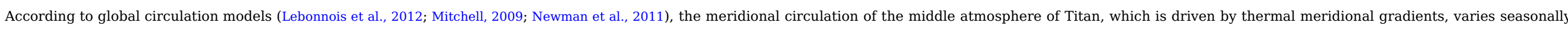

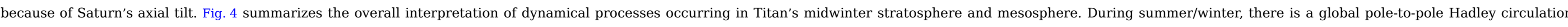

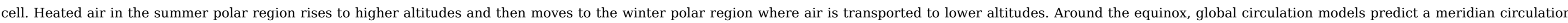

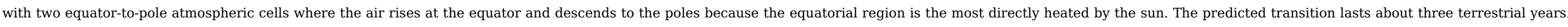

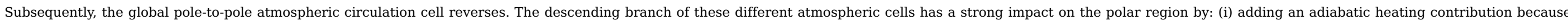

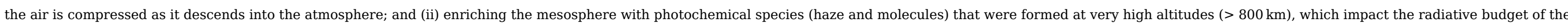
atmosphere and in turn affect the global dynamics. In addition to the main Hadley circulation, a number of these global circulation models find that a small tropospheric cell forms in the summer hemisphere.

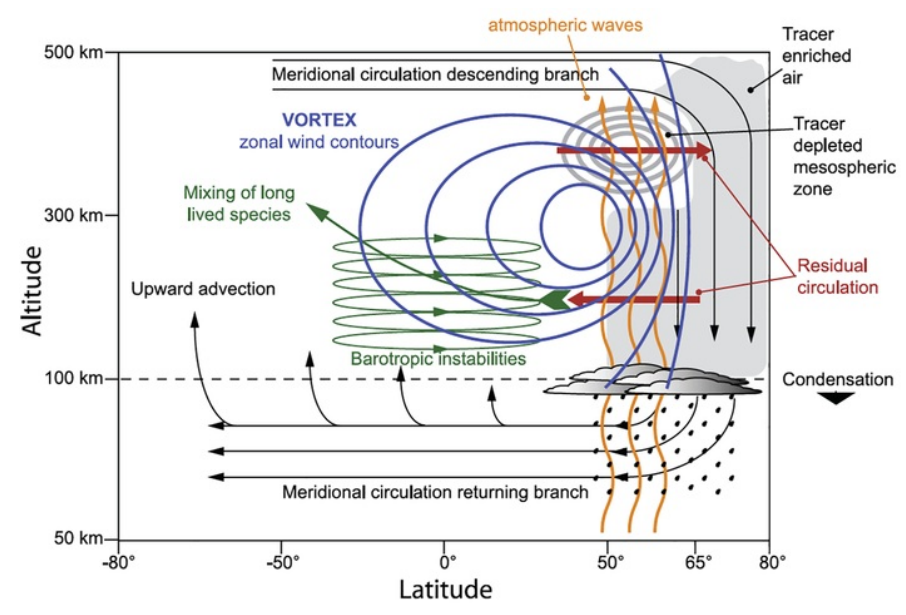

Fig. 4 Schematic of the dynamical processes occurring in Titan's winter polar region.

Reproduced from Teanby NA et al (2008) Titan's winter polar vortex structure revealed by chemical tracers. Journal of Geophysical Research 113(E12). doi: https://doi.org/10.1029/2008JE003218. alt-text: Fig. 4

Zonal winds 


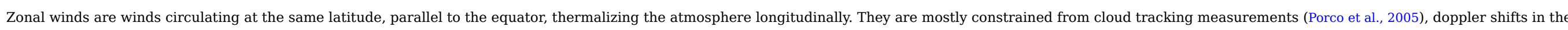

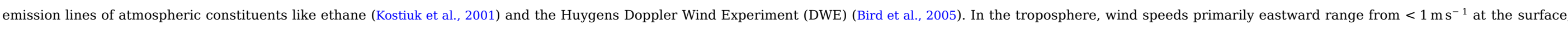

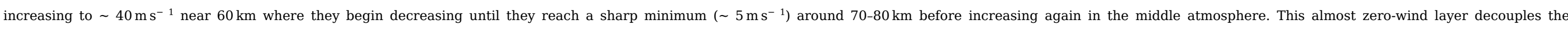

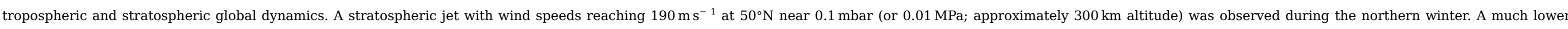

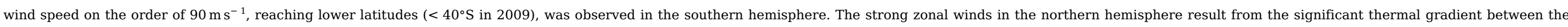

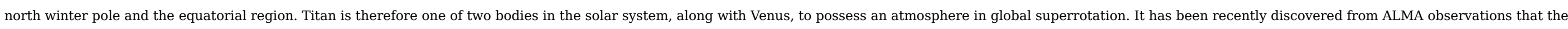
superrotation extends up to at least $1000 \mathrm{~km}$ with surprisingly high wind speeds up to $350 \mathrm{~m} \mathrm{~s}^{-1}$.

\section{Polar vortex}

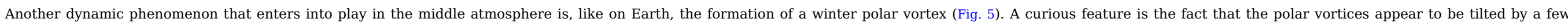

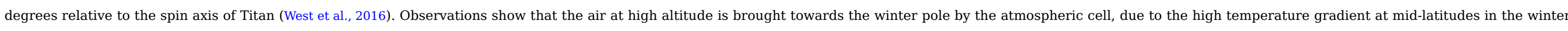

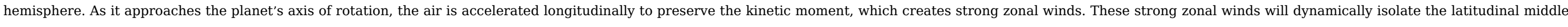

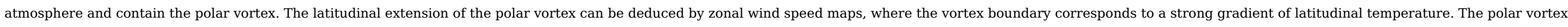
forms during the winter and vanishes in early spring.
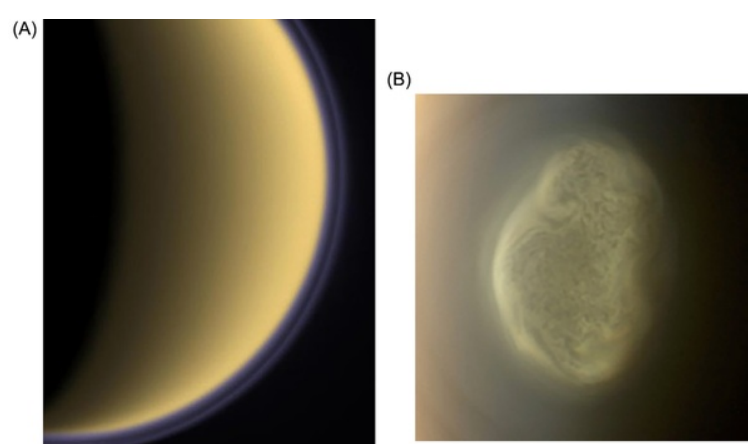

Fig. 5 Left: Titan's thick atmosphere, visible as a thin halo of purple light scattered by small haze particles. A thin, detached haze layer appears to float above the main atmospheric haze. Right: Vortex above Titan's south pole. Credit: NASA, JPL, Space Science Institute.

\section{alt-text: Fig. 5}

\section{Seasonal Change in Composition}

\section{Neutral species}

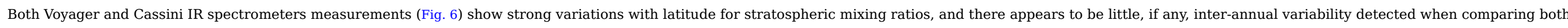

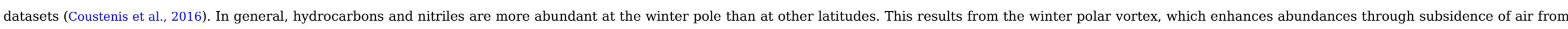

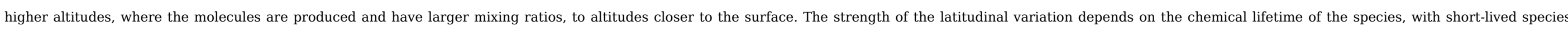

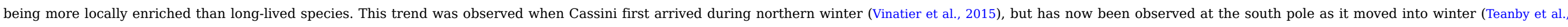

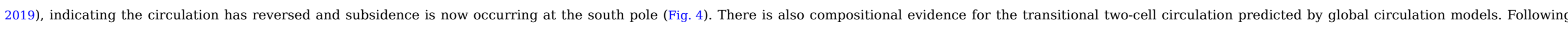

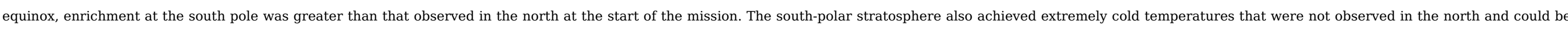

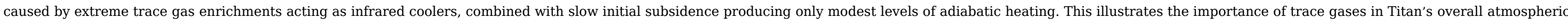
energy budget. 


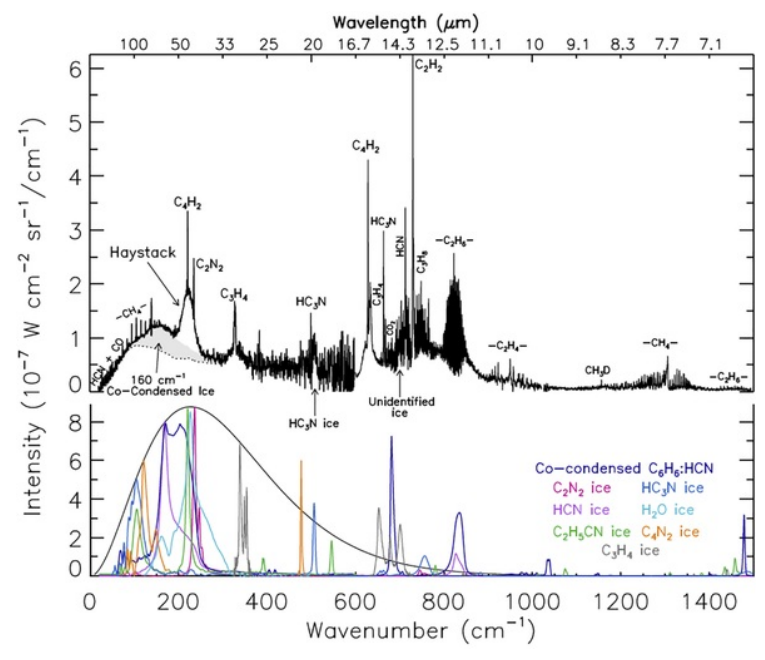

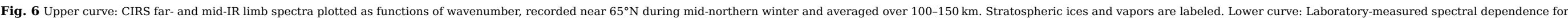
various compounds that may contribute to ice clouds.

Reproduced from Anderson CM, Samuelson RE and Nna-Mvondo D (2018) Organic ices in Titan's stratosphere. Space Science Reviews 214. doi: https://doi.org/10.1007/s11214-018-0559-5.

alt-text: Fig. 6

\section{Haze}

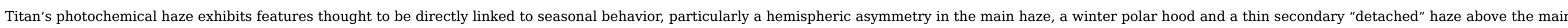
haze layer (Rannou et al., 2012).

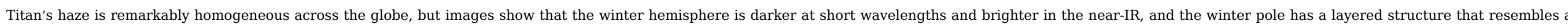

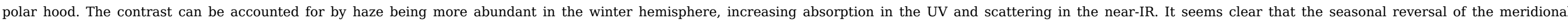
circulation is ultimately responsible for the seasonal variations, with the polar hood being the result of downwelling of haze at the winter pole, but the details remain obscure.

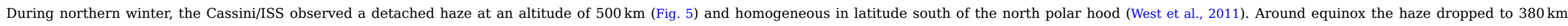

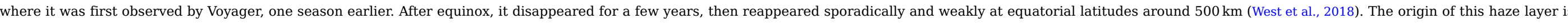

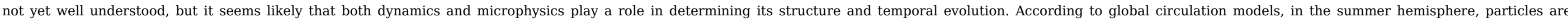

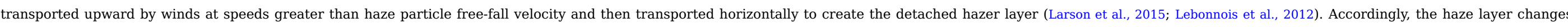

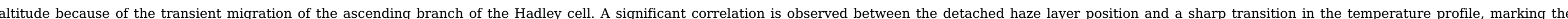
boundary with the thermosphere.

\section{Stratospheric organic ice clouds}

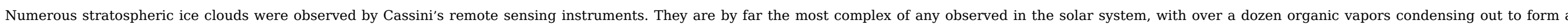

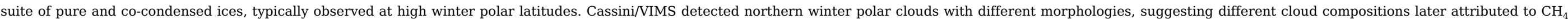

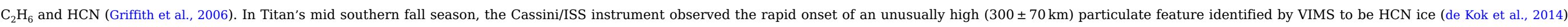

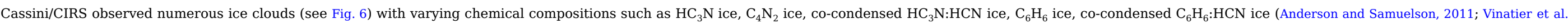

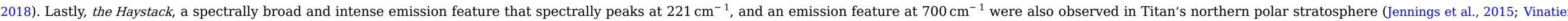

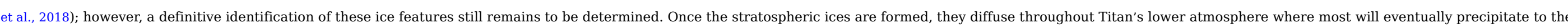




\section{Open Questions}

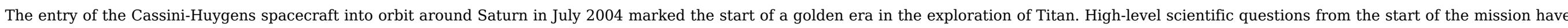
been answered, but others have not been satisfactorily resolved. At the same time, new questions have emerged from the discoveries that have been made, including the following:

Origin and evolution of the atmosphere

- What are the primordial building blocks that lead to such a massive atmosphere, and what can we learn on the solar system formation?

- What are the sources of oxygen in the atmosphere?

- What is controlling the $\mathrm{H}_{2}$ profile and potential spatial variations?

Methane cycle

- Where is methane coming from, and is there a resupply from the interior into the atmosphere at the present day?

- How do clouds form and evolve, and what is the precipitation rate?

- How variable is the weather over long timescales (e.g., years? Decades? Millennia?)?

Chemical processes

- What is the chemical nature of the heavy ions (e.g., $m / z>100$ ), and how do they form?

- What are the composition and optical properties of aerosols in the main haze layer?

- What is the composition and the structure of the massive stratospheric polar clouds?

- How do the haze and various condensates interact?

- To what level of complexity has prebiotic chemistry evolved in the Titan system?

Atmospheric dynamics

- What are the physical processes in the thermosphere that drive the density and temperature variabilities?

- What controls the global dynamics of the upper atmosphere? What are the effects on ion densities?

- What generates (and sustains) Titan's atmospheric superrotation?

- How do the polar vortices form, evolve and terminate?

- What is the wind regime near the surface?

\section{References}

Anderson C.M. and Samuelson R.E., Titan's aerosol and stratospheric ice opacities between 18 and 500 m: Vertical and spectral characteristics from Cassini CIRS, Icarus 212, 2011, 762-778.

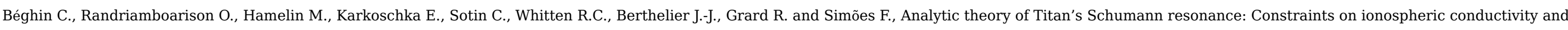
buried water ocean, Icarus 218, 2012, 1028-1042, https://doi.org/10.1016/j.icarus.2012.02.005 


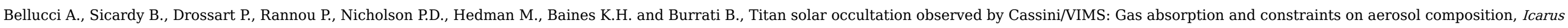
201, 2009, 198-216.

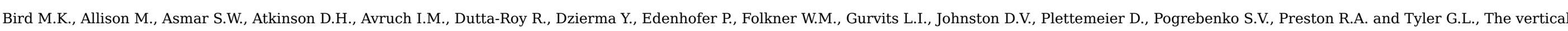
profile of winds on Titan, Nature 438, 2005, 800-802, https://doi.org/10.1038/nature04060.

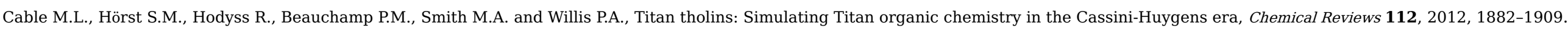

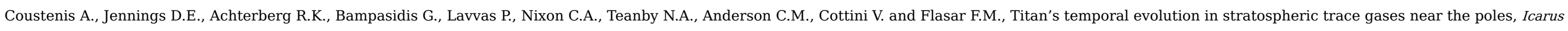
270, 2016, 409-420, https://doi.org/10.1016/j.icarus.2015.08.027.

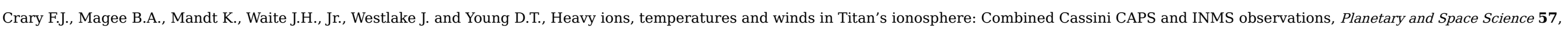
2009, 1847-1856.

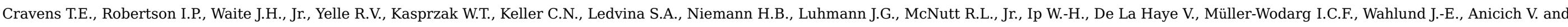
Vuitton V., Composition of Titan's ionosphere, Geophysical Research Letters 33, 2006, L07105.

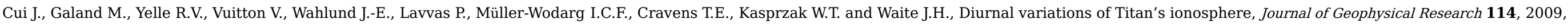
A06310.

de Kok R.J., Teanby N.A., Maltagliati L., Irwin P.G.J. and Vinatier S., HCN ice in Titan's high-altitude southern polar cloud, Nature 514, 2014, 65-67, https://doi.org/10.1038/nature13789.

Fletcher L.N., Orton G.S., Teanby N.A., Irwin P.G.J. and Bjoraker G.L., Methane and its isotopologues on Saturn from Cassini/CIRS observations, Icarus 199, 2009, 351-367,

https://doi.org/10.1016/j.icarus.2008.09.019.

Fortes A.D., Titan's internal structure and the evolutionary consequences, Planetary and Space Science 60, 2012, 10-17, https://doi.org/10.1016/j.pss.2011.04.010.

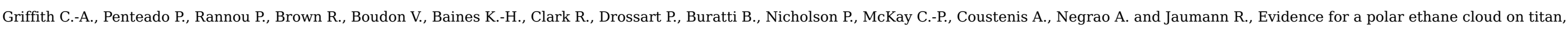
Science 313, 2006, 1620-1622, https://doi.org/10.1126/science.1128245.

Hayes A.G., The lakes and seas of titan, Annual Review of Earth and Planetary Sciences 44, 2016, 57-83, https://doi.org/10.1146/annurev-earth-060115-012247.

Hörst S.M., Titan's atmosphere and climate, Journal of Geophysical Research, Planets 122, 2017, https://doi.org/10.1002/2016JE005240.

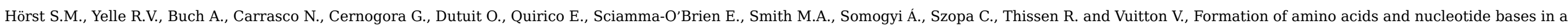
Titan atmosphere simulation experiment, Astrobiology 12, 2012, 809-817.

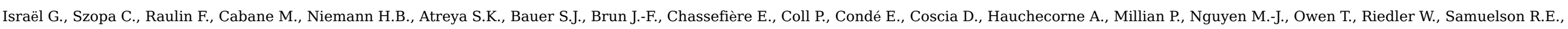
Siguier J.-M., Steller M., Sternberg R. and Vidal-Madjar C., Complex organic matter in Titan's atmospheric aerosols from in situ pyrolysis and analysis, Nature 438, 2005, 796-799.

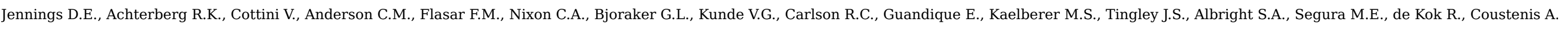

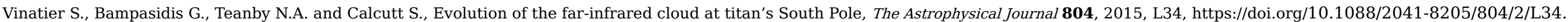

Kostiuk T., Fast K.E., Livengood T.A., Hewagama T., Goldstein J.J., Espenak F. and Buhl D., Direct measurement of winds on Titan, Geophysical Research Letters 28, 2001, 2361-2364, https://doi.org/10.1029/2000GL012617.

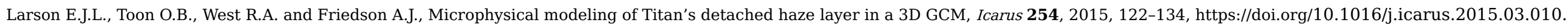

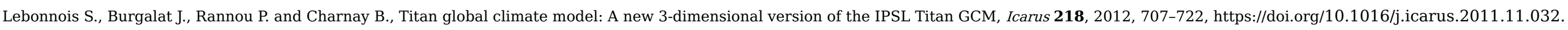




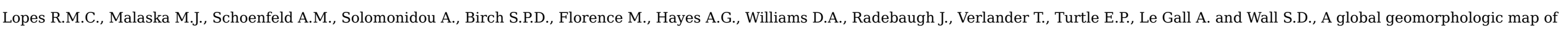
Saturn's moon Titan, Nature Astronomy 4, 2020, 228-233, https://doi.org/10.1038/s41550-019-0917-6.

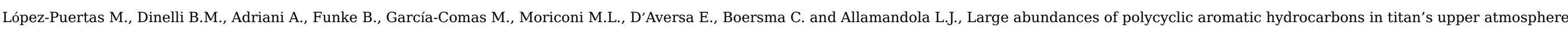
The Astrophysical Journal 770, 2013, 132, https://doi.org/10.1088/0004-637X/770/2/132

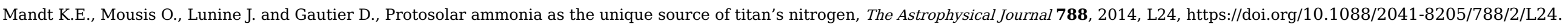

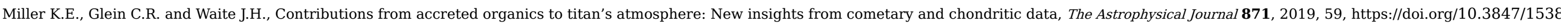
$4357 /$ aaf561.

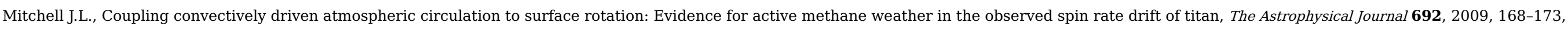
https://doi.org/10.1088/0004-637X/692/1/168.

Newman C.E., Lee C., Lian Y., Richardson M.I. and Toigo A.D., Stratospheric superrotation in the TitanWRF model, Icarus 213, 2011, 636-654, https://doi.org/10.1016/j.icarus.2011.03.025.

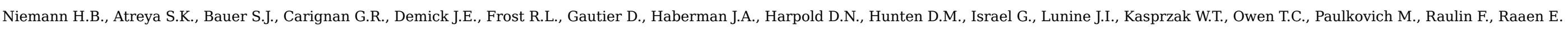
and Way S.H., The abundances of constituents of Titan's atmosphere from the GCMS instrument on the Huygens probe, Nature 438, 2005, 779-784.

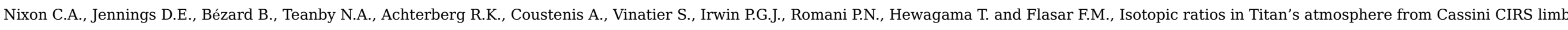
sounding: $\mathrm{CO}_{2}$ at low and midlatitudes, The Astrophysical Journal 681, 2008, L101-L103.

Owen T., Mahaffy P.R., Niemann H.B., Atreya S. and Wong M., Protosolar nitrogen, ApJL 553, 2001, L77.

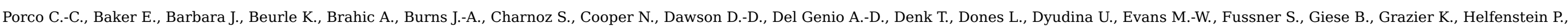

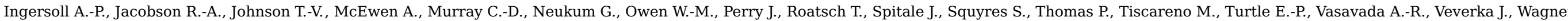
R. and West R., Imaging of Titan from the Cassini spacecraft, Nature 434, 2005, 159-168, https://doi.org/10.1038/nature03436.

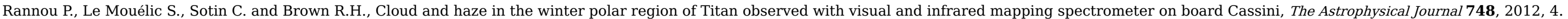

Roe H.G., Titan's methane weather, Annual Review of Earth and Planetary Sciences 40, 2012, 355-382, https://doi.org/10.1146/annurev-earth-040809-152548

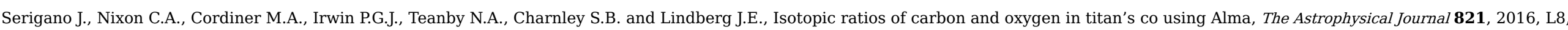
https://doi.org/10.3847/2041-8205/821/1/L8.

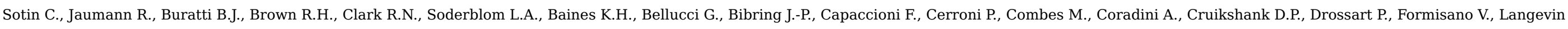

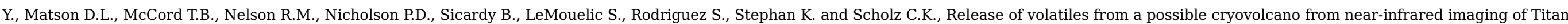
Nature 435, 2005, 786-789, https://doi.org/10.1038/nature03596.

Spilker L., Cassini-Huygens' exploration of the Saturn system: 13 years of discovery, Science 364, 2019, 1046-1051, https://doi.org/10.1126/science.aat3760.

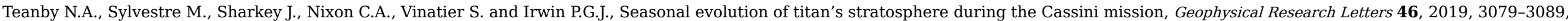
https://doi.org/10.1029/2018GL081401.

Tomasko M.G., Doose L.R., Dafoe L.E. and See C., Limits on the size of aerosols from measurements of linear polarization in Titan's atmosphere, Icarus 204, 2009, 271-283, https://doi.org/10.1016/j.icarus.2009.05.034

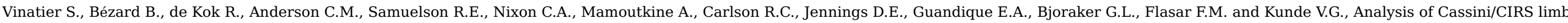
spectra of Titan acquired during the nominal mission II: Aerosol extinction profiles in the 600-1420 cm-1 spectral range, Icarus 210, 2010 , 852-866. 


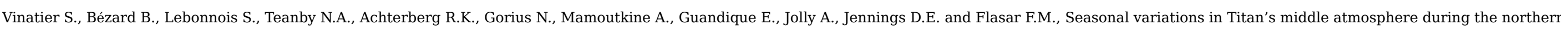
spring derived from Cassini/CIRS observations, Icarus 250, 2015, 95-115, https://doi.org/10.1016/j.icarus.2014.11.019.

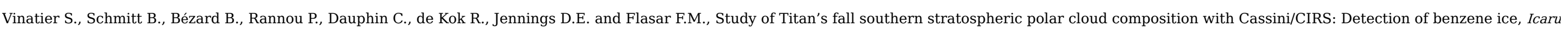
310, 2018, 89-104, https://doi.org/10.1016/j.icarus.2017.12.040.

Vuitton V., Yelle R.V. and Anicich V.G., The nitrogen chemistry of Titan's upper atmosphere revealed, The Astrophysical Journal 647, 2006, L175-L178.

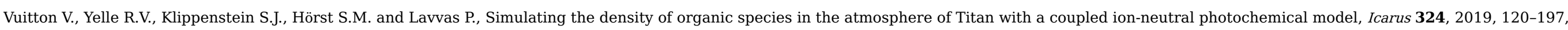
https://doi.org/10.1016/j.icarus.2018.06.013.

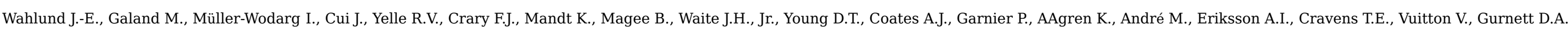
and Kurth W.S., On the amount of heavy molecular ions in Titan's ionosphere, Planetary and Space Science 57, 2009, 1857-1865.

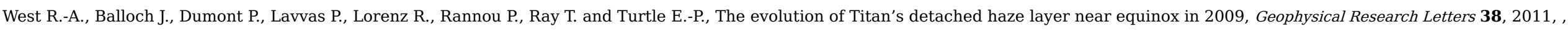
L06204https://doi.org/10.1029/2011GL046843.

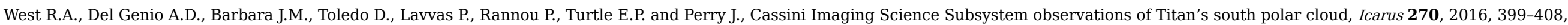
https://doi.org/10.1016/j.icarus.2014.11.038.

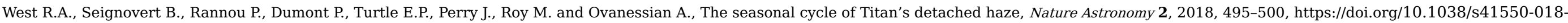
0434-z.

Yung Y.L. and DeMore W.B., Photochemistry of Planetary Atmospheres, 1999, Oxford University Press, Oxford.

Yung Y.L., Allen M. and Pinto J.P., Photochemistry of the atmosphere of Titan: Comparison between model and observations, The Astrophysical Journal Supplement Series 55, 1984, 465-506.

\section{Further Reading}

Anderson C.M., Samuelson R.E. and Nna-Mvondo D., Organic ices in Titan's stratosphere, Space Science Reviews 214, 2018, https://doi.org/10.1007/s11214-018-0559-5.

Bézard B., Yelle R.V. and Nixon C.A., The Composition of Titan's atmosphere, In: Titan: Interior, Surface, Atmosphere and Space Environment, 2014, Cambridge University Press, 158-189.

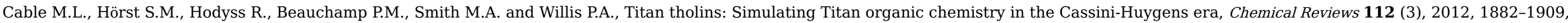
https://doi.org/10.1021/cr200221x.

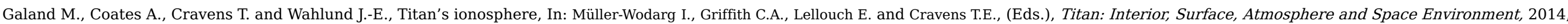
Cambridge University Press, New York, 376-418.

Hörst S.M., Titan's atmosphere and climate, Journal of Geophysical Research, Planets 122, 2017, https://doi.org/10.1002/2016JE005240.

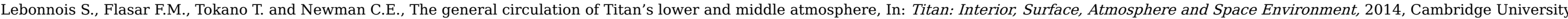
Press, 122-157.

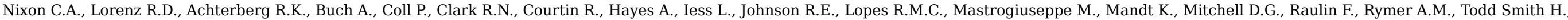

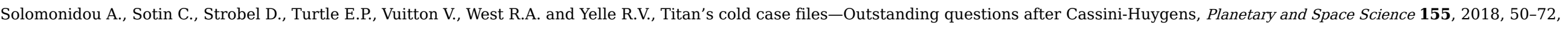
https://doi.org/10.1016/j.pss.2018.02.009.

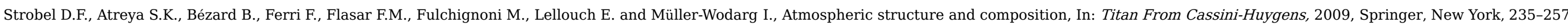




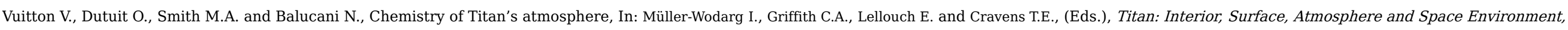
2014, Cambridge University Press, 224-284.

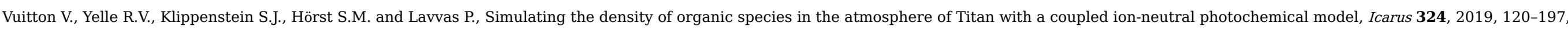
https://doi.org/10.1016/j.icarus.2018.06.013.

West R., Lavvas P., Anderson C. and Imanaka H., Titan's haze, In: Titan: Interior, Surface, Atmosphere and Space Environment, 2014, Cambridge University Press, $285-321$.

\section{Relevant Websites}

https://en.wikipedia.org/wiki/Atmosphere_of_Titan.

https://solarsystem.nasa.gov/moons/saturn-moons/titan/in-depth/.

https://solarsystem.nasa.gov/missions/cassini/overview/.

http://sci.esa.int/cassini-huygens/.

https://www.space.com/15257-titan-saturn-largest-moon-facts-discovery-sdcmp.html.

\section{Biography}

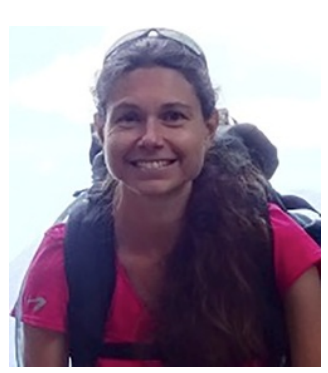

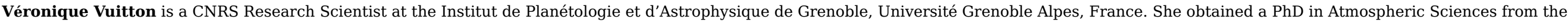

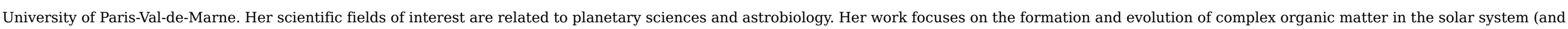

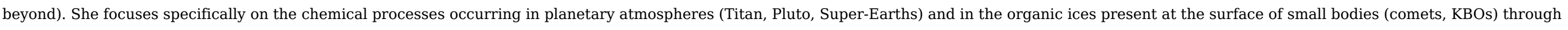
numerical modeling and analytical characterization of laboratory analogs. She was a participating scientist on the Cassini-Huygens mission.

Abstract

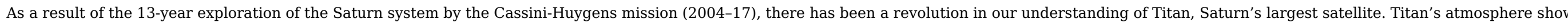

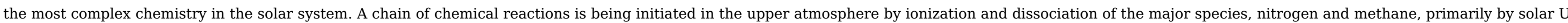

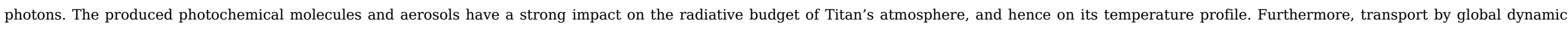

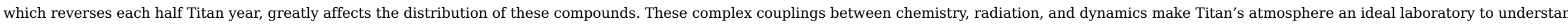

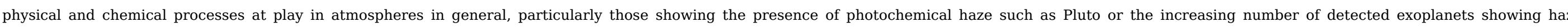

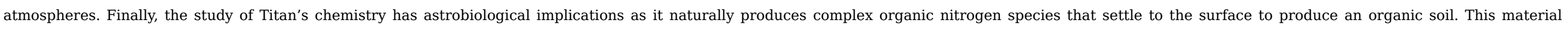

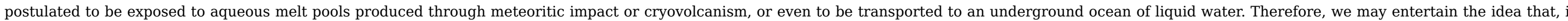
certain regions at the (sub-)surface of Titan, the results of chemistry leading to life as we know it may be present.

Keywords: Aerosol; Atmosphere; Cassini; Chemistry; Clouds; Composition; Dynamics; Huygens; Hydrocarbons; Methane; Nitriles; Nitrogen; Saturnian system; Titan

\section{Queries and Answers}


Query:

Figure 01 is not in printable quality. We are unable to improve at our end. Kindly provide better quality figure.

Answer: The original figure is of poor quality itself. There isn't much I can do about it.

Query:

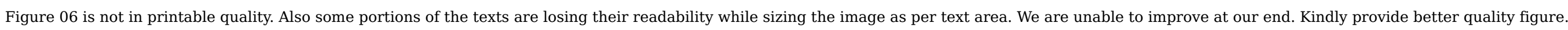
Answer: This is the original figure from the website

Query:

Please check that the affiliations link the authors with their correct departments, institutions, and locations, and correct if necessary.

Answer: The affiliation should be: Univ. Grenoble Alpes, CNRS, CNES, IPAG, 38000 Grenoble, France

Query:

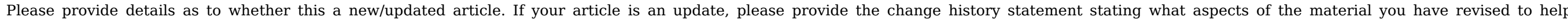
researchers understand what exactly has been updated. An example: "June 2015. VR Meyer updated the text and further readings to this entire article and added new Figure 2".

Answer: This is a new article

Query:

Please provide organization name for all Relevant Websites.

Answer: Wikipedia / NASA / NASA / ESA / Space.com 